\title{
Oscillation and asymptotic behavior of third-order neutral differential equations with distributed deviating arguments
}

\author{
Yazhou Tian ${ }^{1,2^{*}}$, Yuanli Cai ${ }^{1}$, Youliang Fu ${ }^{2}$ and Tongxing $\mathrm{Li}^{2}$
}

\section{"Correspondence:}

tianyazhou369@163.com

'School of Electronic and

Information Engineering, Xi'an

Jiaotong University, Xi'an, Shaanxi 710049, P.R. China

Full list of author information is

available at the end of the article

\begin{abstract}
By employing a generalized Riccati transformation and integral averaging technique, two Philos-type criteria are obtained which ensure that every solution of a class of third-order neutral differential equations with distributed deviating arguments is either oscillatory or converges to zero. These results extend and improve related criteria reported in the literature. Two illustrative examples are provided.
\end{abstract}

MSC: $34 \mathrm{~K} 11$

Keywords: oscillation; asymptotic behavior; third-order neutral differential equation; distributed deviating argument; generalized Riccati transformation

\section{Introduction}

Differential equations with distributed deviating arguments are often used for modeling various problems arising in the engineering and natural sciences. Therefore, analysis of qualitative properties of solutions to such equations is crucial for applications; see Wang [1]. On the basis of these background details, we investigate the oscillation and asymptotic behavior of a third-order neutral differential equation with distributed deviating arguments

$$
\begin{aligned}
& {\left[r(t)\left(\left[x(t)+\int_{a}^{b} p(t, \xi) x(\tau(t, \xi)) d \xi\right]^{\prime \prime}\right)^{\alpha}\right]^{\prime}+\int_{c}^{d} q(t, \xi) f(x(\sigma(t, \xi))) d \xi=0,} \\
& \quad t \geq t_{0},
\end{aligned}
$$

where $\alpha \geq 1$ is the ratio of odd positive integers. Throughout, we suppose that the following assumptions hold.

$\left(\mathrm{A}_{1}\right) \quad r(t) \in C^{1}\left(\left[t_{0}, \infty\right),(0, \infty)\right), r^{\prime}(t) \geq 0, \int_{t_{0}}^{\infty} r^{-1 / \alpha}(s) d s=\infty$;

$\left(\mathrm{A}_{2}\right) p(t, \xi) \in C\left(\left[t_{0}, \infty\right) \times[a, b],[0, \infty)\right), 0 \leq \int_{a}^{b} p(t, \xi) d \xi \leq P<1$;

$\left(\mathrm{A}_{3}\right) \tau(t, \xi) \in C\left(\left[t_{0}, \infty\right) \times[a, b], R\right)$ is a nondecreasing function for $\xi$ satisfying $\tau(t, \xi) \leq t$ and $\liminf _{t \rightarrow \infty} \tau(t, \xi)=\infty$ for $\xi \in[a, b]$;

$\left(\mathrm{A}_{4}\right) q(t, \xi) \in C\left(\left[t_{0}, \infty\right) \times[c, d],[0, \infty)\right)$;

(A $\left.\mathrm{A}_{5}\right) \sigma(t, \xi) \in C\left(\left[t_{0}, \infty\right) \times[c, d], R\right)$ is a nondecreasing function for $\xi$ satisfying $\sigma(t, \xi) \leq t$ and $\liminf _{t \rightarrow \infty} \sigma(t, \xi)=\infty$ for $\xi \in[c, d]$

(c) 2015 Tian et al. This article is distributed under the terms of the Creative Commons Attribution 4.0 International License (http://creativecommons.org/licenses/by/4.0/), which permits unrestricted use, distribution, and reproduction in any medium, provided you give appropriate credit to the original author(s) and the source, provide a link to the Creative Commons license, and indicate if changes were made. 
(A $\left.\mathrm{A}_{6}\right) f(x) \in C(R, R)$ and there exists a positive constant $K$ such that $f(x) / x^{\alpha} \geq K$ for all $x \neq 0$.

Define a new function $z(t)$ by

$$
z(t)=x(t)+\int_{a}^{b} p(t, \xi) x(\tau(t, \xi)) d \xi
$$

By a solution of (1.1) we mean a nontrivial function $x(t) \in C\left(\left[T_{x}, \infty\right), R\right), T_{x} \geq t_{0}$, which has the properties $z(t) \in C^{2}\left(\left[T_{x}, \infty\right), R\right)$ and $r(t)\left(z^{\prime \prime}(t)\right)^{\alpha} \in C^{1}\left(\left[T_{x}, \infty\right), R\right)$ for $T_{x} \geq t_{0}$. Our attention is restricted to those solutions of (1.1) which satisfy $\sup \{|x(t)|: t \geq T\}>0$ for any $T \geq T_{x}$. A solution $x(t)$ of $(1.1)$ is said to be oscillatory on $\left[T_{x}, \infty\right)$ if it is neither eventually positive nor eventually negative. Otherwise it is called nonoscillatory. Equation (1.1) is called oscillatory if all its solutions are oscillatory.

Recently, there has been much research activity concerning the oscillation and asymptotic properties of various classes of differential equations; see, e.g., [1-21] and the references cited therein. So far, there are few results dealing with the asymptotic behavior of third-order neutral differential equations with distributed deviating arguments, we refer the reader to $[17,19]$. The third-order neutral differential equation

$$
\left[r(t)\left([x(t)+p(t) x(\tau(t))]^{\prime \prime}\right)^{\alpha}\right]^{\prime}+q(t) f(x(\sigma(t)))=0
$$

and its special cases have been studied by Baculíková and Džurina [5, 6], Candan [7], Grace et al. [9], Jiang and Li [10], and Li et al. [14]. Using Riccati transformation, Zhang et al. [19] considered a class of third-order neutral differential equations

$$
\left[r(t)\left(x(t)+\int_{a}^{b} p(t, \xi) x(\tau(t, \xi)) d \xi\right)^{\prime \prime}\right]^{\prime}+\int_{c}^{d} q(t, \xi) f(x(\sigma(t, \xi))) d \xi=0
$$

and they obtained several Philos-type (see [15]) criteria for (1.2), whereas Şenel and Utku [17] studied (1.1).

In the study of oscillation of differential equations, there are two techniques which are used to reduce the higher-order equations to the first-order Riccati equations (or inequalities). One of them is the Riccati transformation technique which has been recently extended to dynamic equations on time scales; see, e.g., Şenel and Utku [17]. The other one is termed the generalized Riccati transformation technique; we refer the reader to $\mathrm{Li}$ [11], Li et al. [12], Li and Saker [13], and the related references cited therein. In particular, Li [11] used the generalized Riccati substitution and established several oscillation criteria for a second-order ordinary differential equation

$$
\left(r(t) x^{\prime}(t)\right)^{\prime}+q(t) x(t)=0
$$

Furthermore, he proved that the equation

$$
\left(\frac{1}{t} x^{\prime}(t)\right)^{\prime}+\frac{1}{t^{3}} x(t)=0
$$

is oscillatory and showed that the results established by the Riccati transformation technique cannot be applied. 
In the special case when $\alpha=1,(1.1)$ reduces to (1.2). Now the following question arises. Could we obtain new Philos-type oscillation criteria for (1.1) by using a generalized Riccati transformation which differs from that of [19]? Motivated by Li [11], Li et al. [12], and Li and Saker [13], our purpose in this paper is to give a positive answer to this question. In Section 2, four lemmas are given to prove the main results. In Section 3, we establish two Philos-type theorems for (1.1). In Section 4, two examples and some conclusions are presented to illustrate the main results. As customary, all functional inequalities considered in this paper are supposed to hold for all $t$ large enough.

\section{Some lemmas}

Lemma 2.1 Suppose that conditions $\left(\mathrm{A}_{1}\right)-\left(\mathrm{A}_{6}\right)$ are satisfied and let $x(t)$ be a positive solution of (1.1). Then $z(t)$ has only one of the following two properties:

(I) $z(t)>0, z^{\prime}(t)>0, z^{\prime \prime}(t)>0, z^{\prime \prime \prime}(t) \leq 0$;

(II) $z(t)>0, z^{\prime}(t)<0, z^{\prime \prime}(t)>0, z^{\prime \prime \prime}(t) \leq 0$,

for $t \geq t_{1}$, where $t_{1} \geq t_{0}$ is sufficiently large.

Proof Assume that $x(t)$ is a positive solution of (1.1). Then there exists a $t_{1} \geq t_{0}$ such that, for $t \geq t_{1}$,

$$
x(t)>0, \quad x(\tau(t, \xi))>0, \quad \xi \in[a, b], \quad \text { and } \quad x(\sigma(t, \xi))>0, \quad \xi \in[c, d] .
$$

From (1.1) and the definition of $z(t)$, we have $z(t)>0$ and

$$
\left[r(t)\left(z^{\prime \prime}(t)\right)^{\alpha}\right]^{\prime}=-\int_{c}^{d} q(t, \xi) f(x(\sigma(t, \xi))) d \xi \leq 0 .
$$

Thus $r(t)\left(z^{\prime \prime}(t)\right)^{\alpha}$ is nonincreasing and of one sign. Therefore, $z^{\prime \prime}(t)$ is also of one sign and so we have two possibilities: $z^{\prime \prime}(t)<0$ or $z^{\prime \prime}(t)>0$ for $t \geq t_{2} \geq t_{1}$. We assert that $z^{\prime \prime}(t)>0$ for $t \geq t_{2}$. Otherwise, there exists a constant $M>0$ such that, for $t \geq t_{2}$,

$$
z^{\prime \prime}(t) \leq-M^{\frac{1}{\alpha}} \frac{1}{r^{\frac{1}{\alpha}}(t)}<0
$$

Integrating this inequality from $t_{2}$ to $t$, we obtain

$$
z^{\prime}(t) \leq z^{\prime}\left(t_{2}\right)-M^{\frac{1}{\alpha}} \int_{t_{2}}^{t} \frac{1}{r^{\frac{1}{\alpha}}(s)} d s
$$

Letting $t \rightarrow \infty$ and using $\left(\mathrm{A}_{1}\right)$, we get $\lim _{t \rightarrow \infty} z^{\prime}(t)=-\infty$. Thus $z^{\prime}(t)<0$ eventually. But conditions $z^{\prime \prime}(t)<0$ and $z^{\prime}(t)<0$ imply that $z(t)<0$, which contradicts our assumption $z(t)>0$. Hence, $z^{\prime \prime}(t)>0$ for $t \geq t_{2}$. Furthermore, we have, for $t \geq t_{2}$,

$$
\left[r(t)\left(z^{\prime \prime}(t)\right)^{\alpha}\right]^{\prime}=r^{\prime}(t)\left(z^{\prime \prime}(t)\right)^{\alpha}+\alpha r(t)\left(z^{\prime \prime}(t)\right)^{\alpha-1} z^{\prime \prime \prime}(t) \leq 0
$$

This yields $z^{\prime \prime \prime}(t) \leq 0$ for $t \geq t_{2}$ due to condition $\left(\mathrm{A}_{1}\right)$. Therefore, $z(t)$ has only one of the two properties (I) and (II). This completes the proof. 
Lemma 2.2 Let $x(t)$ be a positive solution of (1.1) and assume that corresponding $z(t)$ has the property (II). If

$$
\int_{t_{0}}^{\infty} \int_{v}^{\infty}\left[\frac{1}{r(u)} \int_{u}^{\infty} \int_{c}^{d} q(s, \xi) d \xi d s\right]^{\frac{1}{\alpha}} d u d v=\infty,
$$

then $\lim _{t \rightarrow \infty} x(t)=0$.

Proof Let $x(t)$ be a positive solution of (1.1). Since $z(t)$ has the property (II), there exists a finite constant $l \geq 0$ such that $\lim _{t \rightarrow \infty} z(t)=l \geq 0$. We prove that $l=0$. Assume now that $l>0$. Then we have $l+\varepsilon>z(t)>l$ for all $\varepsilon>0$. Choose $0<\varepsilon<l(1-P) / P$. It is easy to verify that

$$
\begin{aligned}
x(t) & =z(t)-\int_{a}^{b} p(t, \xi) x(\tau(t, \xi)) d \xi \\
& \geq l-\int_{a}^{b} p(t, \xi) z(\tau(t, \xi)) d \xi \geq l-z(\tau(t, a)) \int_{a}^{b} p(t, \xi) d \xi \\
& \geq l-P(l+\varepsilon)=N(l+\varepsilon)>N z(t),
\end{aligned}
$$

where $N=(l-P(l+\varepsilon)) /(l+\varepsilon)>0$. Using $\left(\mathrm{A}_{6}\right)$ and (2.2), we conclude that

$$
\left[r(t)\left(z^{\prime \prime}(t)\right)^{\alpha}\right]^{\prime} \leq-K N^{\alpha} \int_{c}^{d} q(t, \xi) z^{\alpha}(\sigma(t, \xi)) d \xi
$$

Noting that $z(t)$ has the property (II) and using $\left(\mathrm{A}_{5}\right)$, we have

$$
\left[r(t)\left(z^{\prime \prime}(t)\right)^{\alpha}\right]^{\prime} \leq-K N^{\alpha} z^{\alpha}(\sigma(t, d)) \int_{c}^{d} q(t, \xi) d \xi=-q_{1}(t) z^{\alpha}\left(\sigma_{1}(t)\right)
$$

where $q_{1}(t)=K N^{\alpha} \int_{c}^{d} q(t, \xi) d \xi$ and $\sigma_{1}(t)=\sigma(t, d)$. Integrating inequality (2.3) from $t$ to $\infty$, we obtain

$$
r(t)\left(z^{\prime \prime}(t)\right)^{\alpha} \geq \int_{t}^{\infty} q_{1}(s) z^{\alpha}\left(\sigma_{1}(s)\right) d s .
$$

By virtue of $z\left(\sigma_{1}(t)\right) \geq l$,

$$
z^{\prime \prime}(t) \geq l\left[\frac{1}{r(t)} \int_{t}^{\infty} q_{1}(s) d s\right]^{\frac{1}{\alpha}} .
$$

Integrating inequality (2.4) from $t$ to $\infty$, we have

$$
-z^{\prime}(t) \geq l \int_{t}^{\infty}\left[\frac{1}{r(u)} \int_{u}^{\infty} q_{1}(s) d s\right]^{\frac{1}{\alpha}} d u .
$$

Integrating the latter inequality from $t_{1}$ to $\infty$, we obtain

$$
z\left(t_{1}\right) \geq l \int_{t_{1}}^{\infty} \int_{v}^{\infty}\left[\frac{1}{r(u)} \int_{u}^{\infty} q_{1}(s) d s\right]^{\frac{1}{\alpha}} d u d v,
$$


which contradicts (2.1). Hence $l=0$ and $\lim _{t \rightarrow \infty} z(t)=0$. Then it follows from $0 \leq x(t) \leq$ $z(t)$ that $\lim _{t \rightarrow \infty} x(t)=0$. The proof is complete.

Lemma 2.3 ([5], Lemma 3) Assume that $u(t)>0, u^{\prime}(t)>0$, and $u^{\prime \prime}(t)<0$ for $t \geq t_{0}$. If $\sigma(t) \in C\left(\left[t_{0}, \infty\right),[0, \infty)\right), \sigma(t) \leq t$, and $\lim _{t \rightarrow \infty} \sigma(t)=\infty$, then, for every $\beta \in(0,1)$, there exists a $T_{\beta} \geq t_{0}$ such that, for $t \geq T_{\beta}$,

$$
u(\sigma(t)) \geq \beta \frac{\sigma(t)}{t} u(t)
$$

Lemma 2.4 Assume that $u(t)>0, u^{\prime}(t)>0, u^{\prime \prime}(t)>0$, and $u^{\prime \prime \prime}(t) \leq 0$ for $t \geq t_{0}$. Then, for every $\gamma \in(0,1)$, there exists $a T_{\gamma} \geq t_{0}$ such that, for $t \geq T_{\gamma}$,

$$
u(t) \geq \frac{1}{2} \gamma t u^{\prime}(t)
$$

Proof The proof is similar to that of Baculíková and Džurina ([5], Lemma 4), and hence it is omitted.

\section{Main results}

Let

$$
D=\left\{(t, s) \in R^{2}: t \geq s \geq t_{0}\right\} \quad \text { and } \quad D_{0}=\left\{(t, s) \in R^{2}: t>s \geq t_{0}\right\}
$$

The function $H(t, s) \in C(D, R)$ is said to belong to the class $X$ (denoted by $H \in X$ ) if it satisfies

(i) $H(t, t)=0, t \geq t_{0}, H(t, s)>0,(t, s) \in D_{0}$;

(ii) $\partial H(t, s) / \partial s \leq 0$, there exist $\rho(t) \in C^{1}\left(\left[t_{0}, \infty\right),(0, \infty)\right), b(t) \in C^{1}\left(\left[t_{0}, \infty\right),[0, \infty)\right)$, and $h(t, s) \in C\left(D_{0}, R\right)$ satisfying

$$
-\frac{\partial H(t, s)}{\partial s}=H(t, s)\left[\frac{\rho^{\prime}(s)}{\rho(s)}+(\alpha+1) b^{\frac{1}{\alpha}}(s)\right]+h(t, s) .
$$

Theorem 3.1 Assume that conditions $\left(\mathrm{A}_{1}\right)-\left(\mathrm{A}_{6}\right)$ and (2.1) are satisfied. If there exists a function $H \in X$ such that, for some $\beta \in(0,1)$ and $\gamma \in(0,1)$,

$$
\limsup _{t \rightarrow \infty} \frac{1}{H\left(t, t_{0}\right)} \int_{t_{0}}^{t}\left[H(t, s) \psi(s)-\frac{1}{(\alpha+1)^{\alpha+1}} \frac{\rho(s) r(s)|h(t, s)|^{\alpha+1}}{H^{\alpha}(t, s)}\right] d s=\infty
$$

where $\sigma_{2}(t)=\sigma(t, c)$ and

$$
\begin{aligned}
\psi(t)= & K(1-P)^{\alpha} \rho(t)\left(\frac{1}{2} \beta \gamma \frac{\sigma_{2}^{2}(t)}{t}\right)^{\alpha} \int_{c}^{d} q(t, \xi) d \xi \\
& +\rho(t) r(t) b^{1+\frac{1}{\alpha}}(t)-\rho(t)(r(t) b(t))^{\prime},
\end{aligned}
$$

then every solution $x(t)$ of $(1.1)$ is either oscillatory or satisfies $\lim _{t \rightarrow \infty} x(t)=0$.

Proof Assume that (1.1) has a nonoscillatory solution $x(t)$. Without loss of generality, we may assume that $x(t)$ is an eventually positive solution of (1.1). By Lemma 2.1, we observe that $z(t)$ satisfies either (I) or (II) for $t \geq t_{1}$. We consider each of two cases separately. 
Suppose first that $z(t)$ has the property (I). Then we obtain

$$
\begin{aligned}
x(t) & =z(t)-\int_{a}^{b} p(t, \xi) x(\tau(t, \xi)) d \xi \\
& \geq z(t)-\int_{a}^{b} p(t, \xi) z(\tau(t, \xi)) d \xi \geq z(t)-z(\tau(t, b)) \int_{a}^{b} p(t, \xi) d \xi \\
& \geq\left(1-\int_{a}^{b} p(t, \xi) d \xi\right) z(t) \geq(1-P) z(t) .
\end{aligned}
$$

Using $\left(A_{5}\right),\left(A_{6}\right)$, and (3.3), we have

$$
\begin{aligned}
{\left[r(t)\left(z^{\prime \prime}(t)\right)^{\alpha}\right]^{\prime} } & \leq-K \int_{c}^{d} q(t, \xi) x^{\alpha}(\sigma(t, \xi)) d \xi \\
& \leq-K(1-P)^{\alpha} \int_{c}^{d} q(t, \xi) z^{\alpha}(\sigma(t, \xi)) d \xi \\
& \leq-K(1-P)^{\alpha} z^{\alpha}(\sigma(t, c)) \int_{c}^{d} q(t, \xi) d \xi=-q_{2}(t) z^{\alpha}\left(\sigma_{2}(t)\right),
\end{aligned}
$$

where $q_{2}(t)=K(1-P)^{\alpha} \int_{c}^{d} q(t, \xi) d \xi$ and $\sigma_{2}(t)=\sigma(t, c)$. Define a generalized Riccati transformation $\omega(t)$ by

$$
\omega(t)=\rho(t)\left[\frac{r(t)\left(z^{\prime \prime}(t)\right)^{\alpha}}{\left(z^{\prime}(t)\right)^{\alpha}}+r(t) b(t)\right], \quad t \geq t_{1} .
$$

Then we have $\omega(t)>0$ and

$$
\begin{aligned}
\omega^{\prime}(t) & =\rho^{\prime}(t)\left[\frac{r(t)\left(z^{\prime \prime}(t)\right)^{\alpha}}{\left(z^{\prime}(t)\right)^{\alpha}}+r(t) b(t)\right]+\rho(t)\left[\frac{r(t)\left(z^{\prime \prime}(t)\right)^{\alpha}}{\left(z^{\prime}(t)\right)^{\alpha}}+r(t) b(t)\right]^{\prime} \\
& =\frac{\rho^{\prime}(t)}{\rho(t)} \omega(t)+\rho(t)(r(t) b(t))^{\prime}+\rho(t)\left[\frac{r(t)\left(z^{\prime \prime}(t)\right)^{\alpha}}{\left(z^{\prime}(t)\right)^{\alpha}}\right]^{\prime} \\
& =\frac{\rho^{\prime}(t)}{\rho(t)} \omega(t)+\rho(t)(r(t) b(t))^{\prime}+\rho(t) \frac{\left[r(t)\left(z^{\prime \prime}(t)\right)^{\alpha}\right]^{\prime}}{\left(z^{\prime}(t)\right)^{\alpha}}-\alpha \rho(t) r(t)\left(\frac{z^{\prime \prime}(t)}{z^{\prime}(t)}\right)^{\alpha+1} .
\end{aligned}
$$

By virtue of (3.5), we conclude that

$$
\frac{z^{\prime \prime}(t)}{z^{\prime}(t)}=\frac{1}{r^{\frac{1}{\alpha}}(t)}\left(\frac{\omega(t)}{\rho(t)}-r(t) b(t)\right)^{\frac{1}{\alpha}} .
$$

Combining (3.4), (3.6), and (3.7), we have

$$
\begin{aligned}
\omega^{\prime}(t) \leq & \frac{\rho^{\prime}(t)}{\rho(t)} \omega(t)+\rho(t)(r(t) b(t))^{\prime}-\rho(t) q_{2}(t) \frac{z^{\alpha}\left(\sigma_{2}(t)\right)}{\left(z^{\prime}(t)\right)^{\alpha}} \\
& -\frac{\alpha \rho(t)}{r^{\frac{1}{\alpha}}(t)}\left(\frac{\omega(t)}{\rho(t)}-r(t) b(t)\right)^{1+\frac{1}{\alpha}} .
\end{aligned}
$$

Using Lemma 2.4, for every $\gamma \in(0,1)$, there exists a $T_{\gamma} \geq t_{1}$ such that, for $t \geq T_{\gamma}$,

$$
z\left(\sigma_{2}(t)\right) \geq \frac{1}{2} \gamma \sigma_{2}(t) z^{\prime}\left(\sigma_{2}(t)\right) .
$$


From Lemma 2.3, for every $\beta \in(0,1)$, there exists a $T_{\beta} \geq T_{\gamma}$ such that, for $t \geq T_{\beta}$,

$$
\frac{1}{z^{\prime}(t)} \geq \frac{\beta \sigma_{2}(t)}{t z^{\prime}\left(\sigma_{2}(t)\right)}
$$

Define

$$
A^{*}=\frac{\omega(t)}{\rho(t)} \quad \text { and } \quad B^{*}=r(t) b(t)
$$

Using the inequality (see [13])

$$
\left(A^{*}\right)^{1+\frac{1}{\alpha}}-\left(A^{*}-B^{*}\right)^{1+\frac{1}{\alpha}} \leq\left(B^{*}\right)^{\frac{1}{\alpha}}\left[\left(1+\frac{1}{\alpha}\right) A^{*}-\frac{1}{\alpha} B^{*}\right], \quad A^{*} B^{*} \geq 0, \alpha=\frac{\text { odd }}{\text { odd }} \geq 1
$$

we have

$$
\left(\frac{\omega(t)}{\rho(t)}-r(t) b(t)\right)^{1+\frac{1}{\alpha}} \geq \frac{\omega^{1+\frac{1}{\alpha}}(t)}{\rho^{1+\frac{1}{\alpha}}(t)}+\frac{1}{\alpha}(r(t) b(t))^{1+\frac{1}{\alpha}}-\left(1+\frac{1}{\alpha}\right) \frac{(r(t) b(t))^{\frac{1}{\alpha}}}{\rho(t)} \omega(t) .
$$

Using inequalities (3.8)-(3.11), for $t \geq T \geq T_{\beta}$, we have

$$
\begin{aligned}
\omega^{\prime}(t) \leq & \rho(t)(r(t) b(t))^{\prime}-\rho(t) q_{2}(t)\left(\frac{1}{2} \beta \gamma \frac{\sigma_{2}^{2}(t)}{t}\right)^{\alpha}-\rho(t) r(t) b^{1+\frac{1}{\alpha}}(t) \\
& +\left[\frac{\rho^{\prime}(t)}{\rho(t)}+(\alpha+1) b^{\frac{1}{\alpha}}(t)\right] \omega(t)-\frac{\alpha}{(\rho(t) r(t))^{\frac{1}{\alpha}}} \omega^{1+\frac{1}{\alpha}}(t) \\
= & -\psi(t)+A(t) \omega(t)-B(t) \omega^{1+\frac{1}{\alpha}}(t),
\end{aligned}
$$

where $\psi(t)$ is defined as in (3.2), $A(t)=\left(\rho^{\prime}(t) / \rho(t)\right)+(\alpha+1) b^{1 / \alpha}(t)$, and $B(t)=\alpha /(\rho(t) r(t))^{1 / \alpha}$. Multiplying inequality (3.12) by $H(t, s)$ and integrating the resulting inequality from $T$ to $t$, we have

$$
\begin{aligned}
\int_{T}^{t} H(t, s) \psi(s) d s \leq & \int_{T}^{t} H(t, s)\left(-\omega^{\prime}(s)+A(s) \omega(s)-B(s) \omega^{1+\frac{1}{\alpha}}(s)\right) d s \\
= & H(t, T) \omega(T)+\int_{T}^{t}\left(\frac{\partial H(t, s)}{\partial s}+H(t, s) A(s)\right) \omega(s) d s \\
& -\int_{T}^{t} H(t, s) B(s) \omega^{1+\frac{1}{\alpha}}(s) d s \\
= & H(t, T) \omega(T)-\int_{T}^{t} h(t, s) \omega(s) d s-\int_{T}^{t} H(t, s) B(s) \omega^{1+\frac{1}{\alpha}}(s) d s \\
\leq & H(t, T) \omega(T)+\int_{T}^{t}\left[|h(t, s)| \omega(s)-H(t, s) B(s) \omega^{1+\frac{1}{\alpha}}(s)\right] d s
\end{aligned}
$$

Letting $C=|h(t, s)|, D=H(t, s) B(s)$, and using the inequality (see [13])

$$
C \omega-D \omega^{1+\frac{1}{\alpha}} \leq \frac{\alpha^{\alpha}}{(\alpha+1)^{\alpha+1}} \frac{C^{\alpha+1}}{D^{\alpha}}, \quad D>0,
$$


we obtain

$$
\int_{T}^{t} H(t, s) \psi(s) d s \leq H(t, T) \omega(T)+\int_{T}^{t} \frac{1}{(\alpha+1)^{\alpha+1}} \frac{\rho(s) r(s)|h(t, s)|^{\alpha+1}}{H^{\alpha}(t, s)} d s .
$$

Hence

$$
\frac{1}{H(t, T)} \int_{T}^{t}\left[H(t, s) \psi(s)-\frac{1}{(\alpha+1)^{\alpha+1}} \frac{\rho(s) r(s)|h(t, s)|^{\alpha+1}}{H^{\alpha}(t, s)}\right] d s \leq \omega(T)
$$

for all sufficiently large $t$, which contradicts (3.1).

Assume now that $z(t)$ has the property (II). By Lemma 2.2, we have $\lim _{t \rightarrow \infty} x(t)=0$. The proof is complete.

It may happen that assumption (3.1) in Theorem 3.1 fails to hold. Consequently, Theorem 3.1 cannot be applied. The following theorem provides a new oscillation criterion for (1.1).

Theorem 3.2 Let conditions $\left(\mathrm{A}_{1}\right)-\left(\mathrm{A}_{6}\right)$ and (2.1) be satisfied. Assume that there exists a function $H \in X$ such that

$$
0<\inf _{s \geq t_{0}}\left\{\liminf _{t \rightarrow \infty} \frac{H(t, s)}{H\left(t, t_{0}\right)}\right\} \leq \infty
$$

and

$$
\limsup _{t \rightarrow \infty} \frac{1}{H\left(t, t_{0}\right)} \int_{t_{0}}^{t} \frac{\rho(s) r(s)|h(t, s)|^{\alpha+1}}{H^{\alpha}(t, s)} d s<\infty
$$

hold. If there exists a function $\varphi(t) \in C\left(\left[t_{0}, \infty\right), R\right)$ such that, for all $T \geq t_{0}$,

$$
\limsup _{t \rightarrow \infty} \int_{t_{0}}^{t} \rho^{-\frac{1}{\alpha}}(s) r^{-\frac{1}{\alpha}}(s)\left[\varphi_{+}(s)\right]^{\frac{\alpha+1}{\alpha}} d s=\infty
$$

and

$$
\limsup _{t \rightarrow \infty} \frac{1}{H(t, T)} \int_{T}^{t}\left[H(t, s) \psi(s)-\frac{1}{(\alpha+1)^{\alpha+1}} \frac{\rho(s) r(s)|h(t, s)|^{\alpha+1}}{H^{\alpha}(t, s)}\right] d s \geq \varphi(T),
$$

where $\psi(t)$ is defined by $(3.2)$ and $\varphi_{+}(t)=\max \{\varphi(t), 0\}$, then the conclusion of Theorem 3.1 remains intact.

Proof Assuming that $z(t)$ has the property (I) and proceeding as in the proof of Theorem 3.1, we have (3.13) and (3.14) for all $t>T$. Hence, by virtue of (3.14),

$$
\limsup _{t \rightarrow \infty} \frac{1}{H(t, T)} \int_{T}^{t}\left[H(t, s) \psi(s)-\frac{1}{(\alpha+1)^{\alpha+1}} \frac{\rho(s) r(s)|h(t, s)|^{\alpha+1}}{H^{\alpha}(t, s)}\right] d s \leq \omega(T)
$$

for all $t>T$. Thus, by (3.18) and (3.19), we have

$$
\varphi(T) \leq \omega(T)
$$


and

$$
\limsup _{t \rightarrow \infty} \frac{1}{H(t, T)} \int_{T}^{t} H(t, s) \psi(s) d s \geq \varphi(T) .
$$

From (3.20), we obtain

$$
\int_{T}^{\infty} \rho^{-\frac{1}{\alpha}}(s) r^{-\frac{1}{\alpha}}(s) \omega^{\frac{\alpha+1}{\alpha}}(s) d s \geq \int_{T}^{\infty} \rho^{-\frac{1}{\alpha}}(s) r^{-\frac{1}{\alpha}}(s)\left[\varphi_{+}(s)\right]^{\frac{\alpha+1}{\alpha}} d s
$$

and hence, by (3.17),

$$
\int_{T}^{\infty} \rho^{-\frac{1}{\alpha}}(s) r^{-\frac{1}{\alpha}}(s) \omega^{\frac{\alpha+1}{\alpha}}(s) d s=\infty
$$

To complete the proof, we show that (3.23) does not hold. Let

$$
u(t)=\frac{\alpha}{H(t, T)} \int_{T}^{t} H(t, s) \rho^{-\frac{1}{\alpha}}(s) r^{-\frac{1}{\alpha}}(s) \omega^{\frac{\alpha+1}{\alpha}}(s) d s
$$

and

$$
v(t)=\frac{1}{H(t, T)} \int_{T}^{t}|h(t, s)| \omega(s) d s
$$

for all $t>T$. It follows from (3.13) and (3.21) that

$$
\begin{aligned}
\liminf _{t \rightarrow \infty}[u(t)-v(t)] & \leq \omega(T)-\limsup _{t \rightarrow \infty} \frac{1}{H(t, T)} \int_{T}^{t} H(t, s) \psi(s) d s \\
& \leq \omega(T)-\varphi(T)<\infty
\end{aligned}
$$

Now by (3.15), there exists a positive constant $\xi_{1}$ satisfying

$$
\inf _{s \geq t_{0}}\left\{\liminf _{t \rightarrow \infty} \frac{H(t, s)}{H\left(t, t_{0}\right)}\right\}>\xi_{1}>0
$$

Let $\xi_{2}$ be an any positive constant. It follows from (3.23) that, for all $t \geq T_{1}$,

$$
\int_{T}^{t} \rho^{-\frac{1}{\alpha}}(s) r^{-\frac{1}{\alpha}}(s) \omega^{\frac{\alpha+1}{\alpha}}(s) d s \geq \frac{\xi_{2}}{\alpha \xi_{1}}
$$

where $T_{1}>T$ is sufficiently large. Therefore, for all $t \geq T_{1}$,

$$
\begin{aligned}
u(t) & =\frac{\alpha}{H(t, T)} \int_{T}^{t} H(t, s) d\left(\int_{T}^{s} \rho^{-\frac{1}{\alpha}}(\zeta) r^{-\frac{1}{\alpha}}(\zeta) \omega^{\frac{\alpha+1}{\alpha}}(\zeta) d \zeta\right) \\
& \geq \frac{\alpha}{H(t, T)} \int_{T_{1}}^{t}\left(-\frac{\partial H(t, s)}{\partial s}\right)\left(\int_{T}^{s} \rho^{-\frac{1}{\alpha}}(\zeta) r^{-\frac{1}{\alpha}}(\zeta) \omega^{\frac{\alpha+1}{\alpha}}(\zeta) d \zeta\right) d s \\
& \geq \frac{\xi_{2}}{\xi_{1} H(t, T)} \int_{T_{1}}^{t}\left(-\frac{\partial H(t, s)}{\partial s}\right) d s=\frac{\xi_{2} H\left(t, T_{1}\right)}{\xi_{1} H(t, T)} \geq \frac{\xi_{2} H\left(t, T_{1}\right)}{\xi_{1} H\left(t, t_{0}\right)} .
\end{aligned}
$$


By (3.25), there exists a $T_{2} \geq T_{1}$ such that $H\left(t, T_{1}\right) / H\left(t, t_{0}\right) \geq \xi_{1}$ for all $t \geq T_{2}$. Thus $u(t) \geq \xi_{2}$ for all $t \geq T_{2}$. Since $\xi_{2}$ is an arbitrary constant,

$$
\lim _{t \rightarrow \infty} u(t)=\infty
$$

Consider now a sequence $\left\{t_{i}\right\}_{i=1}^{\infty}$ in $(T, \infty)$ with $\lim _{i \rightarrow \infty} t_{i}=\infty$ such that

$$
\lim _{i \rightarrow \infty}\left[u\left(t_{i}\right)-v\left(t_{i}\right)\right]=\liminf _{t \rightarrow \infty}[u(t)-v(t)]<\infty
$$

By virtue of (3.24), there exist a natural number $N_{0}$ and a constant $L>0$ such that, for all $i>N_{0}$,

$$
u\left(t_{i}\right)-v\left(t_{i}\right) \leq L
$$

It follows from (3.26) that

$$
\lim _{i \rightarrow \infty} u\left(t_{i}\right)=\infty
$$

Combining (3.27) and (3.28), we conclude that

$$
\lim _{i \rightarrow \infty} v\left(t_{i}\right)=\infty
$$

and, for $i$ large enough,

$$
\frac{v\left(t_{i}\right)}{u\left(t_{i}\right)}>\frac{1}{2}
$$

From (3.29) and (3.30), we obtain

$$
\lim _{i \rightarrow \infty} \frac{v^{\alpha+1}\left(t_{i}\right)}{u^{\alpha}\left(t_{i}\right)}=\infty
$$

On the other hand, by Hölder's inequality, we have

$$
\begin{aligned}
v\left(t_{i}\right) \leq & \left\{\frac{\alpha}{H\left(t_{i}, T\right)} \int_{T}^{t_{i}} H\left(t_{i}, s\right) \rho^{-\frac{1}{\alpha}}(s) r^{-\frac{1}{\alpha}}(s) \omega^{\frac{\alpha+1}{\alpha}}(s) d s\right\}^{\frac{\alpha}{\alpha+1}} \\
& \times\left\{\frac{1}{\alpha^{\alpha} H\left(t_{i}, T\right)} \int_{T}^{t_{i}} \frac{\rho(s) r(s)\left|h\left(t_{i}, s\right)\right|^{\alpha+1}}{H^{\alpha}\left(t_{i}, s\right)} d s\right\}^{\frac{1}{\alpha+1}} .
\end{aligned}
$$

Therefore, for all $i$ large enough,

$$
\frac{v^{\alpha+1}\left(t_{i}\right)}{u^{\alpha}\left(t_{i}\right)} \leq \frac{1}{\alpha^{\alpha} \xi_{1} H\left(t_{i}, t_{0}\right)} \int_{t_{0}}^{t_{i}} \frac{\rho(s) r(s)\left|h\left(t_{i}, s\right)\right|^{\alpha+1}}{H^{\alpha}\left(t_{i}, s\right)} d s .
$$

From (3.31) and (3.32), we deduce that

$$
\lim _{i \rightarrow \infty} \frac{1}{H\left(t_{i}, t_{0}\right)} \int_{t_{0}}^{t_{i}} \frac{\rho(s) r(s)\left|h\left(t_{i}, s\right)\right|^{\alpha+1}}{H^{\alpha}\left(t_{i}, s\right)} d s=\infty,
$$


so

$$
\lim _{t \rightarrow \infty} \frac{1}{H\left(t, t_{0}\right)} \int_{t_{0}}^{t} \frac{\rho(s) r(s)|h(t, s)|^{\alpha+1}}{H^{\alpha}(t, s)} d s=\infty
$$

which contradicts (3.16). Therefore, (3.23) cannot hold. By virtue of (3.22), we get

$$
\int_{T}^{\infty} \rho^{-\frac{1}{\alpha}}(s) r^{-\frac{1}{\alpha}}(s)\left[\varphi_{+}(s)\right]^{\frac{\alpha+1}{\alpha}} d s<\infty
$$

which contradicts (3.17).

Suppose that $z(t)$ has the property (II). By Lemma 2.2, we obtain $\lim _{t \rightarrow \infty} x(t)=0$. This completes the proof.

\section{Examples and conclusions}

Example 4.1 For $t \geq 1$, consider a third-order differential equation

$$
\left[t^{2}\left(\left[x(t)+\int_{\frac{1}{2}}^{1} \frac{4 \xi}{3 t^{2}} x\left(\frac{t+\xi}{3}\right) d \xi\right]^{\prime \prime}\right)^{3}\right]^{\prime}+\int_{0}^{1} \frac{32 q_{0} \xi}{t^{5}} x^{3}\left(\frac{t+\xi}{2}\right) d \xi=0
$$

where $q_{0}>0$ is a constant. Let $\alpha=3, a=1 / 2, b=1, c=0, d=1, r(t)=t^{2}, p(t, \xi)=4 \xi /\left(3 t^{2}\right)$, $\tau(t, \xi)=(t+\xi) / 3, q(t, \xi)=32 q_{0} \xi / t^{5}$, and $\sigma(t, \xi)=(t+\xi) / 2$. Then

$$
\int_{a}^{b} p(t, \xi) d \xi=\int_{\frac{1}{2}}^{1} \frac{4 \xi}{3 t^{2}} d \xi=\frac{1}{2 t^{2}} \leq \frac{1}{2} \quad \text { and } \quad \sigma_{2}(t)=\sigma(t, 0)=\frac{t}{2}
$$

It is not difficult to verify that

$$
\int_{1}^{\infty} \frac{1}{s^{\frac{2}{3}}} d s=\infty \text { and } \int_{1}^{\infty} \int_{v}^{\infty}\left[\frac{1}{u^{2}} \int_{u}^{\infty} \int_{0}^{1} \frac{32 q_{0} \xi}{s^{5}} d \xi d s\right]^{\frac{1}{3}} d u d v=\infty
$$

Therefore, the conditions $\left(\mathrm{A}_{1}\right)-\left(\mathrm{A}_{6}\right)$ and (2.1) are satisfied. Furthermore, we choose $K=1$, $P=1 / 2, \rho(t)=t, b(t)=0$, and $H(t, s)=(t-s)^{4}$. Then $h(t, s)=(t-s)^{3}\left(5-t s^{-1}\right)$,

$$
\psi(t)=\left(1-\frac{1}{2}\right)^{3} t\left(\frac{\beta \gamma\left(\frac{t}{2}\right)^{2}}{2 t}\right)^{3} \int_{0}^{1} \frac{32 q_{0} \xi}{t^{5}} d \xi=\frac{q_{0}(\beta \gamma)^{3}}{256 t}
$$

and

$$
\begin{gathered}
\limsup _{t \rightarrow \infty} \frac{1}{H\left(t, t_{0}\right)} \int_{t_{0}}^{t}\left[H(t, s) \psi(s)-\frac{1}{(\alpha+1)^{\alpha+1}} \frac{\rho(s) r(s)|h(t, s)|^{\alpha+1}}{H^{\alpha}(t, s)}\right] d s \\
=\limsup _{t \rightarrow \infty} \frac{1}{(t-1)^{4}} \int_{1}^{t}\left[\frac{q_{0}(\beta \gamma)^{3}}{256}(t-s)^{4} \frac{1}{s}-\frac{1}{256} s^{3}\left(5-t s^{-1}\right)^{4}\right] d s \\
=\limsup _{t \rightarrow \infty} \frac{1}{(t-1)^{4}} \int_{1}^{t}\left[\frac{q_{0}(\beta \gamma)^{3}}{256}\left(t^{4} s^{-1}-4 t^{3}+6 t^{2} s-4 t s^{2}+s^{3}\right)\right. \\
\left.-\left(\frac{1}{256} t^{4} s^{-1}-\frac{5}{64} t^{3}+\frac{75}{128} t^{2} s-\frac{125}{64} t s^{2}+\frac{625}{256} s^{3}\right)\right] d s=\infty,
\end{gathered}
$$


if $q_{0}>1 /(\beta \gamma)^{3}$ for some $\beta \in(0,1)$ and $\gamma \in(0,1)$. Hence, by Theorem 3.1, every solution $x(t)$ of (4.1) is either oscillatory or converges to zero as $t \rightarrow \infty$ in the case where $q_{0}>$ $1,000,000 /(531,441) \approx 1.9$ (by letting $\beta=\gamma=9 / 10)$. Observe that the results reported in [19] cannot be applied to (4.1) since $\alpha=3$.

Example 4.2 For $t \geq 1$, consider a third-order differential equation

$$
\left[x(t)+\int_{\frac{1}{2}}^{1} \frac{4 \xi}{3 t^{2}} x\left(\frac{t+\xi}{3}\right) d \xi\right]^{\prime \prime \prime}+\int_{0}^{1} \frac{16 q_{0} \xi}{t^{3}} x\left(\frac{t+\xi}{2}\right) d \xi=0,
$$

where $q_{0}>0$ is a constant. Let $\alpha=1, a=1 / 2, b=1, c=0, d=1, r(t)=1, p(t, \xi)=4 \xi /\left(3 t^{2}\right)$, $\tau(t, \xi)=(t+\xi) / 3, q(t, \xi)=16 q_{0} \xi / t^{3}$, and $\sigma(t, \xi)=(t+\xi) / 2$. Then

$$
\int_{a}^{b} p(t, \xi) d \xi=\int_{\frac{1}{2}}^{1} \frac{4 \xi}{3 t^{2}} d \xi=\frac{1}{2 t^{2}} \leq \frac{1}{2} \quad \text { and } \quad \sigma_{2}(t)=\sigma(t, 0)=\frac{t}{2} .
$$

It is easy to verify that

$$
\int_{1}^{\infty} \frac{1}{r(s)} d s=\infty \text { and } \int_{1}^{\infty} \int_{v}^{\infty}\left[\int_{u}^{\infty} \int_{0}^{1} q(s, \xi) d s\right] d u d v=\infty
$$

Therefore, the conditions $\left(\mathrm{A}_{1}\right)-\left(\mathrm{A}_{6}\right)$ and (2.1) are satisfied. Furthermore, we choose $K=1$, $P=1 / 2, \rho(t)=t, b(t)=1 / t$, and $H(t, s)=(t-s)^{2}$. Then $h(t, s)=(t-s)\left(5-3 t s^{-1}\right)$,

$$
\psi(t)=\left(1-\frac{1}{2}\right) t\left(\frac{\beta \gamma\left(\frac{t}{2}\right)^{2}}{2 t}\right) \int_{0}^{1} \frac{16 q_{0} \xi}{t^{3}} d \xi+t\left(\frac{1}{t}\right)^{2}-t\left(\frac{1}{t}\right)^{\prime}=\left(\frac{1}{2} q_{0} \beta \gamma+2\right) \frac{1}{t}
$$

and

$$
\begin{aligned}
& \limsup _{t \rightarrow \infty} \frac{1}{H\left(t, t_{0}\right)} \int_{t_{0}}^{t}\left[H(t, s) \psi(s)-\frac{1}{(\alpha+1)^{\alpha+1}} \frac{\rho(s) r(s)|h(t, s)|^{\alpha+1}}{H^{\alpha}(t, s)}\right] d s \\
& \quad=\limsup _{t \rightarrow \infty} \frac{1}{(t-1)^{2}} \int_{1}^{t}\left[\left(\frac{1}{2} q_{0} \beta \gamma+2\right)(t-s)^{2} \frac{1}{s}-\frac{1}{4} s\left(5-3 t s^{-1}\right)^{2}\right] d s \\
& \quad=\limsup _{t \rightarrow \infty} \frac{1}{(t-1)^{2}} \int_{1}^{t}\left[\left(\frac{1}{2} q_{0} \beta \gamma+2\right)\left(t^{2} s^{-1}-2 t+s\right)-\frac{1}{4}\left(25 s-30 t+9 t^{2} s^{-1}\right)\right] d s \\
& \quad=\infty
\end{aligned}
$$

if $q_{0}>1 /(2 \beta \gamma)$ for some $\beta \in(0,1)$ and $\gamma \in(0,1)$. Therefore, by Theorem 3.1, every solution $x(t)$ of (4.2) is either oscillatory or converges to zero as $t \rightarrow \infty$ in the case where $q_{0}>$ $50 / 81 \approx 0.62$ (by letting $\beta=\gamma=9 / 10$ ).

Remark 4.1 With an appropriate choice of the function $H$, one can derive from Theorems 3.1 and 3.2 a number of oscillation criteria for (1.1). For example, consider a Kamenev-type function $H(t, s)$ by $H(t, s)=(t-s)^{n-1},(t, s) \in D$, where $n>2$ is an integer. The remainder of the details are left to the reader.

Remark 4.2 Theorems 3.1 and 3.2 reported in this paper reduce to ([19], Theorems 3.1 and 3.2), respectively, when letting $\alpha=1$ and $b(t)=0$. 
Remark 4.3 Note that Theorems 3.1 and 3.2 ensure that every solution $x(t)$ to (1.1) is either oscillatory or satisfies $\lim _{t \rightarrow \infty} x(t)=0$ and, unfortunately, these results cannot distinguish solutions with different behaviors. Since the sign of the derivative $z^{\prime}(t)$ is not fixed, it is not easy to establish sufficient conditions which guarantee that all solutions to (1.1) are just oscillatory and do not satisfy $\lim _{t \rightarrow \infty} x(t)=0$. Neither is it possible to use the technique exploited in this paper for proving that all solutions of (1.1) satisfy $\lim _{t \rightarrow \infty} x(t)=0$. Hence, these two interesting problems are left for future research.

Remark 4.4 It would be interesting to find a different method to investigate (1.1) when $0<\alpha<1$. It would also be of interest to find another method to study (1.1) in the case where $\int_{t_{0}}^{\infty} r^{-1 / \alpha}(s) d s<\infty$.

Competing interests

The authors declare that they have no competing interests.

Authors' contributions

All four authors contributed equally to this work. They all read and approved the final version of the manuscript.

\section{Author details}

${ }^{1}$ School of Electronic and Information Engineering, Xi'an Jiaotong University, Xi'an, Shaanxi 710049, P.R. China. ${ }^{2}$ Qingdao Technological University, Feixian, Shandong 273400, P.R. China.

\section{Acknowledgements}

The authors are grateful to the editors and two anonymous referees for a very thorough reading of the manuscript and for pointing out several inaccuracies. This research was supported by NNSF of P.R. China (Grant Nos. 61174217, 61374074, and 61473133) and NSF of Shandong Province (Grant No. JQ201119).

Received: 23 February 2015 Accepted: 11 August 2015 Published online: 28 August 2015

\section{References}

1. Wang, PG: Oscillation criteria for second-order neutral equations with distributed deviating arguments. Comput. Math. Appl. 47, 1935-1946 (2004)

2. Agarwal, RP, Grace, SR, O'Regan, D: Oscillation Theory for Difference and Functional Differential Equations. Kluwer Academic, Dordrecht (2000)

3. Agarwal, RP, Grace, SR, O'Regan, D: Oscillation Theory for Second Order Dynamic Equations. Taylor \& Francis, London (2003)

4. Aktaş, MF, Tiryaki, A, Zafer, A: Oscillation criteria for third-order nonlinear functional differential equations. Appl. Math. Lett. 23, 756-762 (2010)

5. Baculíková, B, Džurina, J: Oscillation of third-order neutral differential equations. Math. Comput. Model. 52, 215-226 (2010)

6. Baculíková, B, Džurina, J: Oscillation of third-order functional differential equations. Electron. J. Qual. Theory Differ. Equ. 2010, 43 (2010)

7. Candan, T: Asymptotic properties of solutions of third-order nonlinear neutral dynamic equations. Adv. Differ. Equ. 2014, 35 (2014)

8. Candan, T: Oscillation criteria and asymptotic properties of solutions of third-order nonlinear neutral differential equations. Math. Methods Appl. Sci. 38, 1379-1392 (2015)

9. Grace, SR, Agarwal, RP, Pavani, R, Thandapani, E: On the oscillation of certain third order nonlinear functional differential equations. Appl. Math. Comput. 202, 102-112 (2008)

10. Jiang, Y, Li, T: Asymptotic behavior of a third-order nonlinear neutral delay differential equation. J. Inequal. Appl. 2014, $512(2014)$

11. Li, HJ: Oscillation criteria for second order linear differential equations. J. Math. Anal. Appl. 194, $217-234$ (1995)

12. Li, T, Rogovchenko, YuV, Zhang, C: Oscillation results for second-order nonlinear neutral differential equations. Adv. Differ. Equ. 2013, 336 (2013)

13. Li, T, Saker, SH: A note on oscillation criteria for second-order neutral dynamic equations on isolated time scales. Commun. Nonlinear Sci. Numer. Simul. 19,4185-4188 (2014)

14. Li, T, Zhang, C, Xing, G: Oscillation of third-order neutral delay differential equations. Abstr. Appl. Anal. 2012, Article ID 569201 (2012). doi:10.1155/2012/569201

15. Philos, ChG: Oscillation theorems for linear differential equations of second order. Arch. Math. 53, 482-492 (1989)

16. Rogovchenko, YuV: Oscillation theorems for second-order equations with damping. Nonlinear Anal. 41, 1005-1028 (2000)

17. Senel, MT, Utku, N: Oscillation criteria for third-order neutral dynamic equations with continuously distributed delay. Adv. Differ. Equ. 2014, 220 (2014)

18. Tiryaki, A, Aktaş, MF: Oscillation criteria of a certain class of third order nonlinear delay differential equations with damping. J. Math. Anal. Appl. 325, 54-68 (2007) 
19. Zhang, QX, Gao, L, Yu, YH: Oscillation criteria for third-order neutral differential equations with continuously distributed delay. Appl. Math. Lett. 25, 1514-1519 (2012)

20. Wang, $\mathrm{PG}, \mathrm{Cai}, \mathrm{H}$ : Oscillatory criteria for higher order functional differential equations with damping. J. Funct. Spaces Appl. 2013, Article ID 968356 (2013). doi:10.1155/2013/968356

21. Erbe, LH, Kong, Q, Zhang, BG: Oscillation Theory for Functional Differential Equations. Dekker, New York (1995)

Submit your manuscript to a SpringerOpen ${ }^{\odot}$ journal and benefit from:

- Convenient online submission

- Rigorous peer review

- Immediate publication on acceptance

- Open access: articles freely available online

- High visibility within the field

- Retaining the copyright to your article

Submit your next manuscript at $\boldsymbol{s p r i n g e r o p e n . c o m ~}$ 